\title{
Magnetotelluric source effect due to 3D ionospheric current systems using the complex image method for 1D conductivity structures
}

\author{
Ari Viljanen, Risto Pirjola, and Olaf Amm \\ Finnish Meteorological Institute, Geophysical Research Division, P.O. Box 503, FIN-00101 Helsinki, Finland \\ (Received December 14, 1998; Revised September 3, 1999; Accepted September 16, 1999)
}

\begin{abstract}
The complex image method (CIM) is an efficient tool to calculate the electromagnetic field at the earth's surface produced by 3D ionospheric current systems when the earth has a layered conductivity structure. The calculations are applicable to the estimation of source effects on magnetotelluric data. In this paper CIM is used in connection with some typical high-latitude ionospheric events: a westward travelling surge, a Harang discontinuity, an omega band, and a giant pulsation. The complicated ionospheric current systems are constructed of short horizontal current filaments with vertical currents at both ends. The currents are given numerically on a $50 \mathrm{~km} \times 50 \mathrm{~km}$ grid covering a region of even $1000 \mathrm{~km} \times 2000 \mathrm{~km}$. The investigations indicate that the source distortion very much depends on the event, and may be significant in a wide period range, especially for a resistive earth structure. The source effect seems quite unpredictable. Sometimes the apparent resistivity is larger and sometimes smaller than the plane wave value. At times the source effect is very small even if the ionospheric current is strongly inhomogeneous.
\end{abstract}

\section{Introduction}

The usual assumption in magnetotelluric studies of the earth's conductivity structure is that the primary field produced by ionospheric-magnetospheric currents is laterally uniform, i.e. the plane wave criterion is satisfied. Generally this is the case except for areas near the auroral and equatorial electrojets where the source effect distortion may seriously hamper the interpretation of data (e.g. Mareschal, 1986; Osipova et al., 1989; Viljanen, 1996; Padilha et al., 1997). A careful selection of events to be analyzed may decrease the source distortion, and robust techniques to deal with data contaminated by source effects have also been developed and applied (e.g. Chave et al., 1987; Egbert and Booker, 1989a,b; Viljanen et al., 1993; Larsen et al., 1996; Garcia et al., 1997). However, the source currents constitute a complicated 3D system which changes from event to event in an unpredictable way.

Häkkinen and Pirjola (1986) derived exact formulas for the electromagnetic field at the surface of a 1-D, layered earth assuming a 3D ionospheric-magnetospheric current system. However, the expressions are complicated inverse Fourier transforms and thus impractical in any time-critical applications. Pirjola (1992) and Viljanen et al. (1993) demonstrate the use of the 3D model for magnetotelluric source effect investigations.

The calculation of the surface fields is greatly simplified and accelerated if the contribution of a layered earth is represented by an image of the primary ionospheric source. This technique generally leads to a complex image depth, which can be regarded just as a mathematical trick. However, the

Copy right $($ C The Society of Geomagnetism and Earth, Planetary and Space Science (SGEPSS); The Seismological Society of Japan; The Volcanological Society of Japan; The Geodetic Society of Japan; The Japanese Society for Planetary Sciences. real and imaginary parts also imply the depths of the in-phase and out-of-phase telluric currents (Weidelt, 1972; Szarka and Fischer, 1989). The use of the complex image method (CIM) was suggested by Wait and Spies (1969), and reconsidered by Boteler and Pirjola (1998). Applying the study by Thomson and Weaver (1975), Pirjola and Viljanen (1998) extended the CIM concept to the case of a straight horizontal current of a finite length over a layered earth. Vertical currents at both ends of this current are determined by the condition that the total current is divergence-free.

The assumption of strictly vertical currents is reasonable in the auroral region where the inclination is close to 90 degrees (Amm, 1995). The extension of the image method is based on the fact that vertical currents are equivalent to a horizontal current distribution as concerns the magnetic field and the total horizontal electric field at the earth's surface.

Any ionospheric current distribution can be superposed of the above-described elementary current filaments lying at specified grid points. So the results by Pirjola and Viljanen (1998) permit the treatment of any current system. CIM is also an excellent tool in studies of geomagnetically induced currents (GIC) in technological systems (Pirjola et al., 1999), where the knowledge of the electric field is crucial.

In this paper we apply CIM to the calculation of the electromagnetic field at the earth's surface in several realistic cases: a westward travelling surge (WTS), a Harang discontinuity, an omega band, and a giant pulsation. For comparison, we also consider a plane wave and a line current of a finite length. This paper is also a preparatory work for analyses of the data collected in the Baltic Electromagnetic Array Research (BEAR) project (Korja, 1998). 


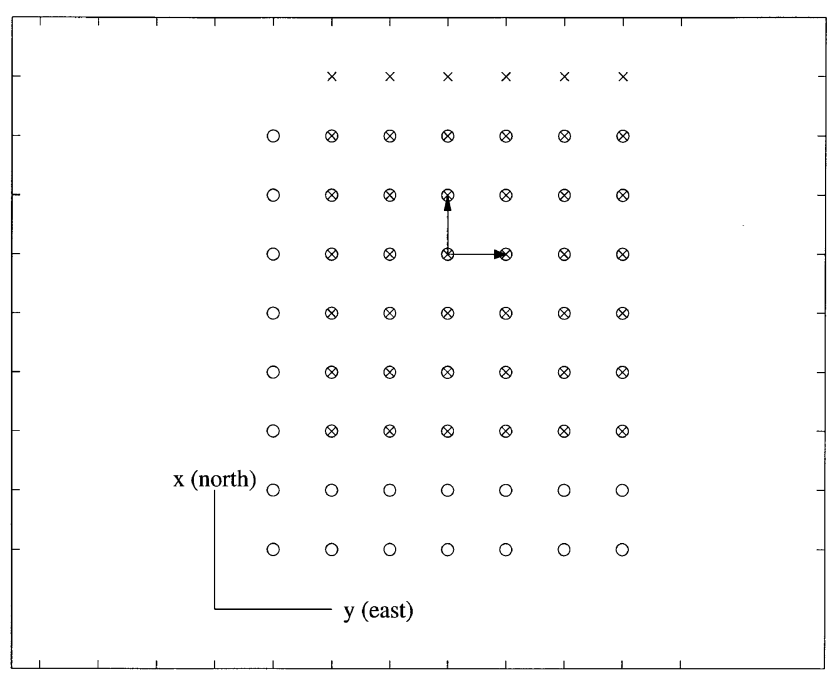

Fig. 1. Synchronized ionospheric (marked with " $\bigcirc$ ”) and earth surface ("X") grids (top view). Two horizontal current elements are shown. Vertical currents start or end at the grid points. In this paper, $x$ points to the (geographic) north, $y$ east, $z$ downwards, and the earth's surface is the $x y$ plane.

\section{CIM Algorithm}

We construct the ionospheric current system of short straight horizontal filaments with vertical currents at both ends. The most practical way is to use northward and eastward elements set on a rectangular grid. The field is calculated at the earth's surface on another grid, which can be defined independently of the ionospheric grid. However, computations become faster if the surface grid is synchronized with the ionospheric one as in Fig. 1. The elements are set in such way on the grid that the vertical currents are at the grid points. In this paper the ionospheric and earth grids have the same spacing. Thus, any grid point in the ionosphere can be reached through a multiple shift (measured in the grid spacing) of any earth grid point. The coordinate system is the standard one with $x$ pointing northward, $y$ eastward and $z$ downward. The earth's surface is the $x y$ plane, and the ionospheric plane lies at $z=-h$. The field formulas for an eastward elementary current are given in the Appendix.

The amplitude of the current is given as a time series for each element. To be able to use CIM formulas, we apply FFT (fast Fourier transform). For each frequency, the complex image depth is determined by the surface impedance, and the electromagnetic field is obtained from analytical formulas. All current elements are treated in the same way, and the total field is the sum of their fields. The time domain field is obtained by the inverse FFT.

\section{Numerical Results}

The ionospheric current models applied here are determined by using ground magnetic data and partly also ionospheric radar observations (Amm, 1995, 1996). Two different layered earth models, representing a conductive and a resistive structure, are considered (Table 1). The models are adopted from Viljanen and Pirjola (1994), and a conductive basement starting at the depth of $150 \mathrm{~km}$ is added in both. Although these models are based on earlier studies in Finland,
Table 1. Conductive and resistive earth models. The cumulative conductance is given at the bottom of each layer.

\begin{tabular}{lcc}
\hline & Conductive model & \\
Depth $[\mathrm{km}]$ & Resistivity $[\Omega \mathrm{m}]$ & Conductance $[1 / \Omega]$ \\
\hline $0-3$ & 5000 & 0.6 \\
$3-9$ & 500 & 12.6 \\
$9-14$ & 100 & 63 \\
$14-21$ & 10 & 763 \\
$21-44$ & 20 & 1912 \\
$44-150$ & 1000 & 2018 \\
$150-$ & 1 & \\
\hline & Resistive model & \\
Depth $[\mathrm{km}]$ & Resistivity $[\Omega \mathrm{m}]$ & Conductance $[1 / \Omega]$ \\
\hline $0-12$ & 30000 & 0.4 \\
$12-34$ & 3000 & 7.7 \\
$34-50$ & 50 & 321 \\
$50-100$ & 1000 & 370 \\
$100-150$ & 5000 & 380 \\
$150-$ & 1 & \\
\hline
\end{tabular}

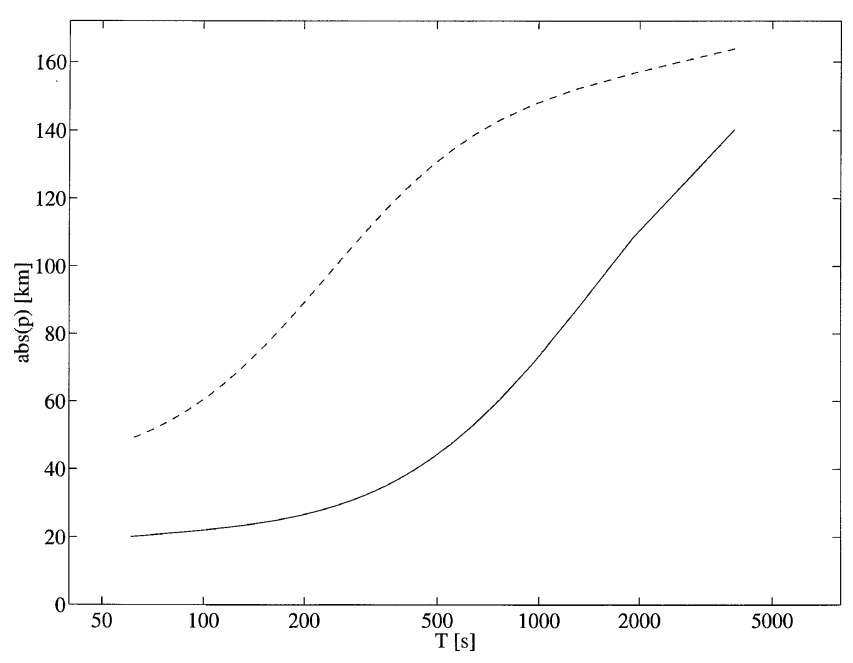

Fig. 2. Absolute value of the complex skin depth. Uniform line: conductive earth model, dashed line: resistive model.

they are only used here as examples of two fairly different cases. The corresponding complex skin depths are shown in Fig. 2.

We will use the following definitions of the complex apparent resistivities $\rho_{x x}, \rho_{y y}, \rho_{x y}$ and $\rho_{y x}$ :

$$
\begin{aligned}
& \rho_{x x}(\omega, x, y)=-\frac{i \mu_{0}}{\omega}\left(\frac{E_{x}(\omega, x, y)}{B_{x}(\omega, x, y)}\right)^{2} \\
& \rho_{y y}(\omega, x, y)=-\frac{i \mu_{0}}{\omega}\left(\frac{E_{y}(\omega, x, y)}{B_{y}(\omega, x, y)}\right)^{2}
\end{aligned}
$$



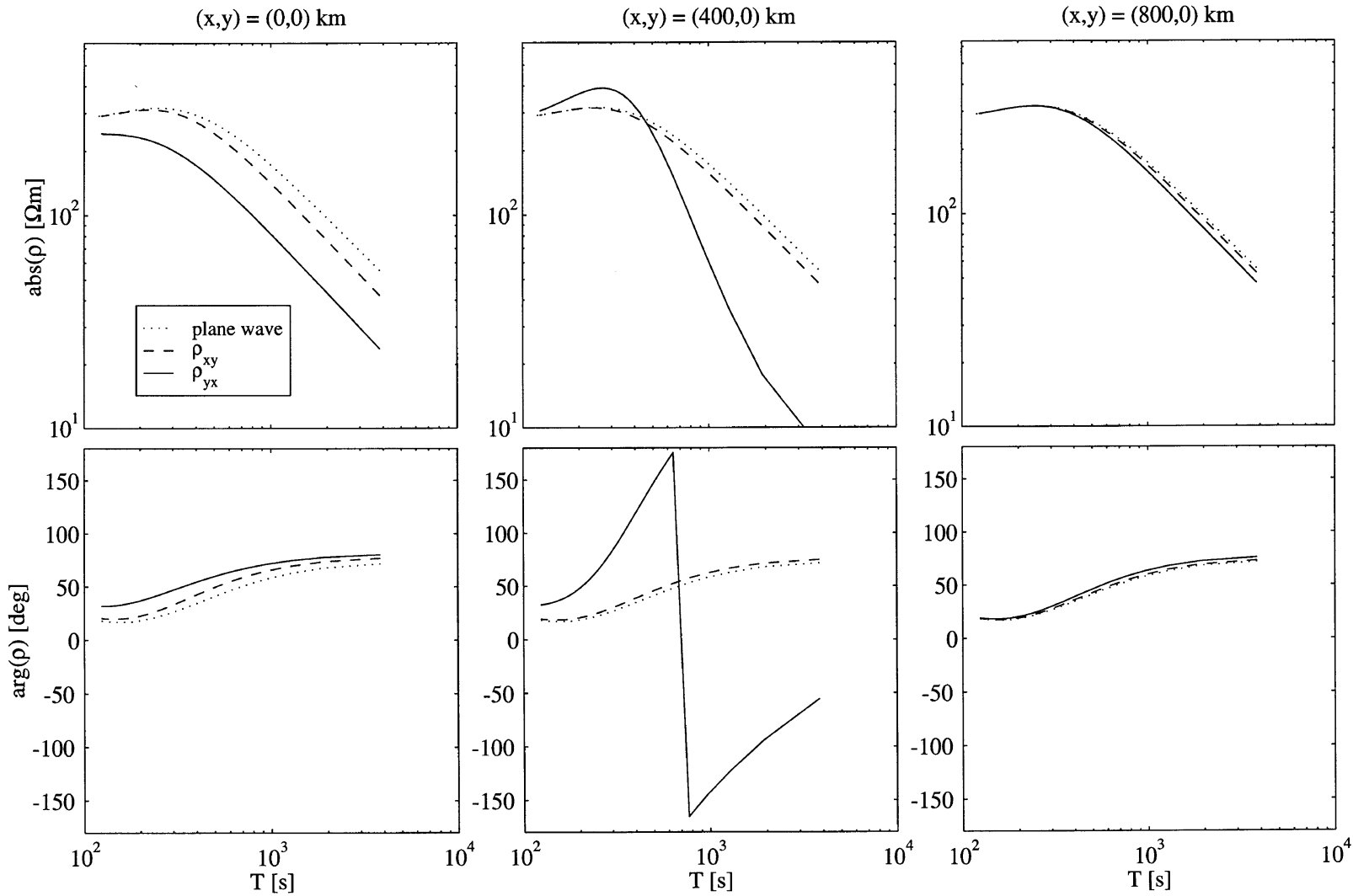

Fig. 3. Absolute values and phases of the apparent resistivities $\rho_{x y}$ and $\rho_{y x}$ as a function of period at $x=0,400,800 \mathrm{~km}(y=0 \mathrm{~km})$ in the case of a line current flowing above the $y$ axis at the height of $110 \mathrm{~km}$ from $(x, y)=(0,-500) \mathrm{km}$ to $(x, y)=(0,500) \mathrm{km}$ with vertical currents at the ends. The resistive earth model is used. The plane wave value is plotted as a dotted line.

$$
\begin{aligned}
& \rho_{x y}(\omega, x, y)=-\frac{i \mu_{0}}{\omega}\left(\frac{E_{x}(\omega, x, y)}{B_{y}(\omega, x, y)}\right)^{2} \\
& \rho_{y x}(\omega, x, y)=-\frac{i \mu_{0}}{\omega}\left(\frac{E_{y}(\omega, x, y)}{B_{x}(\omega, x, y)}\right)^{2} .
\end{aligned}
$$

In the plane wave case $\rho_{x y}$ and $\rho_{y x}$ are equal.

In the following subsections we briefly outline the ionospheric current models, and show the apparent resistivity curves. We start with the electrojet model as a basic reference. In all cases the plane wave result is given too.

\subsection{Electrojet}

Consider a horizontal line current flowing above the $y$ axis from $(x, y)=(0,-500) \mathrm{km}$ to $(x, y)=(0,500) \mathrm{km}$, with vertical currents at both ends. The horizontal currents are assumed to flow at the height of $110 \mathrm{~km}$ throughout this paper. In all examples, the grid spacing is $50 \mathrm{~km}$.

Although the model is an oversimplification, some basic results concerning the apparent resistivity still remain qualitatively valid in many cases. Figure 3 shows the apparent resistivities $\rho_{x y}$ and $\rho_{y x}$ at sites $x=0,400,800 \mathrm{~km}(y=0$ $\mathrm{km})$ for the resistive earth model. All four apparent resistivities are plotted as contour maps in Fig. 4. The conductive model yields qualitatively similar results, but the source distortion is generally smaller.

In the vicinity of the current $(x=0), \rho_{y x}$ is smaller than the plane wave value, then larger $(x=400 \mathrm{~km})$, and finally approaches the plane wave value $(x=800 \mathrm{~km})$. Large maxima are found around about $x= \pm 400 \mathrm{~km}$, where $\rho_{y x}$ can be several times larger than the plane wave value. This is due to the change of sign of $B_{x}$ near these points, making the denominator in Eq. (1) very small. The zero points are caused by the opposite signs of $B_{x}$ due to the horizontal filament and the vertical currents. The sign reversal is often seen in observed data of long meridional magnetometer chains across the auroral region (e.g. Untiedt and Baumjohann, 1993), and the length of the electrojet considered here has a reasonable value.

The apparent resistivity $\rho_{x y}$ is quite smooth, but $\rho_{x x}$ and $\rho_{y y}$ are very peculiar even in this simple model. For example, $\rho_{x x}$ is exactly zero along the $y$ axis because $E_{x}$ vanishes there.

We also checked the accuracy of CIM by considering an infinitely long line current and comparing $\rho_{y x}$ calculated with CIM and from exact integral formulas. For both earth models used here the coindidence is excellent in the period range $100 \ldots 10000 \mathrm{~s}$ and at distances $0 \ldots 1000 \mathrm{~km}$ from the current. The largest relative errors in the magnitude are about $10 \%$.

\subsection{Westward travelling surge}

The westward travelling surge (WTS) is an intense current occurring in connection with auroral breakups before and around local midnight (Fig. 5). The main parts are a westward current which turns upward in the head of WTS near the origin, where there is also an intense counterclockwize current vortex. The whole system moves westward with a velocity of 1-10 km/s; here we consider the velocity of $1 \mathrm{~km} / \mathrm{s}$. We assume that the current system of Fig. 5 

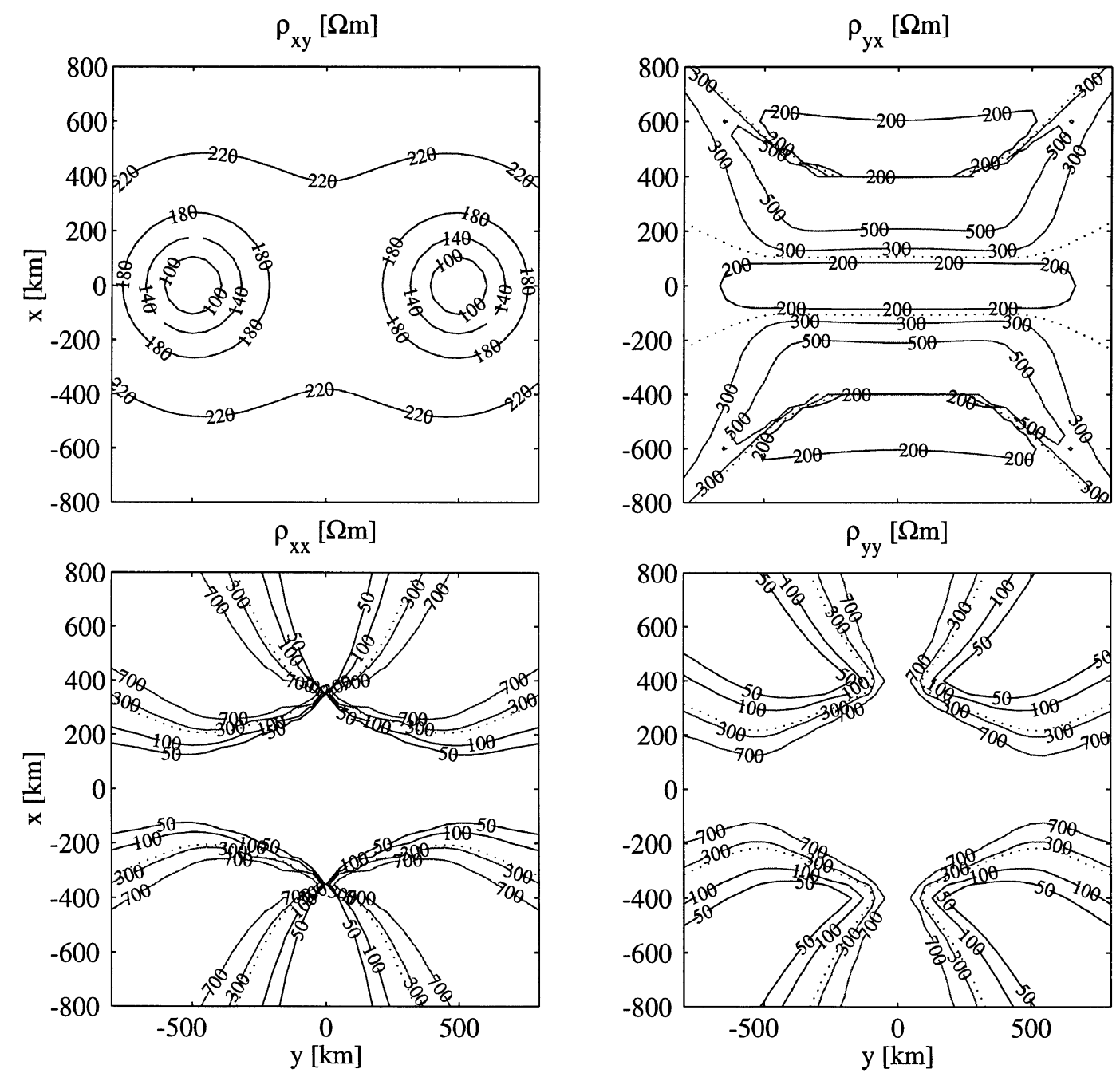

Fig. 4. Absolute values of the apparent resistivities $\rho_{x y}$ and $\rho_{y x}$ (upper panel), and $\rho_{x x}$ and $\rho_{y y}$ (lower panel) as a contour map for the electrojet model in Fig. 3. The period is $640 \mathrm{~s}$ and the plane wave value of the apparent resistivity is $237 \Omega \mathrm{m}$ (dotted line).

is constant in the frame which moves with WTS. The time dependence then results from the motion of WTS over the ionospheric grid in Fig. 1. Around $x=0 \mathrm{~km} B_{x}$ is charaterized by a negative peak, and $B_{y}$ by first a positive and then a smaller negative excursion.

In all models hereafter we have slightly extended the horizontal current distribution to avoid unrealistically large vertical currents at the boundaries. For clarity, these extensions are not shown in the current model plots. Outside the extended grid all currents are assumed to be zero.

Figure 6 displays the $x$ component of the magnetic field disturbance and the $y$ component of the associated electric field in the time domain along the profile $y=0 \mathrm{~km}$. For clarity, we have zoomed in the region where the variation is largest. The head of WTS crosses the profile at about 01:20. The currents in Fig. 5 are scaled to yield quite an intense variation at the earth's surface.

We have smoothed the apparent resistivity curves, because otherwize they are very spiky due to very small values of the electric or magnetic field at some sites (the plane wave curves are not modified). The absolute value at a given period $T$ is computed by first taking the average of $|E|^{2}$ and $|B|^{2}$ in the period range $(T-\Delta T, T+\Delta T)$, and then applying Eq. (1). $\Delta T$ used here is half of the shortest period available. Figures 7 and 8 show the apparent resistivities $\rho_{x y}$ and $\rho_{y x}$ at sites $x=0,400,800 \mathrm{~km}$ on the profile $y=0 \mathrm{~km}$, assuming the conductive and resistive earth models, respectively.

Although the resistivity curves are here and there quite intricate, their basic behaviour is in many cases the same as that of the electrojet: near the current centre the values are smaller, then at long periods larger, and at large distances equal to the plane wave result (cf. $\rho_{y x}$ in Fig. 4). The complicated results are expected because even the simple electrojet model produces spatially strongly varying resistivities. In the WTS model the field at one point is remarkably affected by some tens of nearby current elements, which may have opposite signs and very different amplitudes.

Furthermore, we have not performed any preprocessing on the (simulated) data time series. A standard magnetotelluric analysis would probably remove the worst outliers in apparent resistivity curves. For example, Chave et al. (1987) show how a robust technique produces much smoother power spectra (and consequently transfer functions) than conventional methods, especially at short periods. 


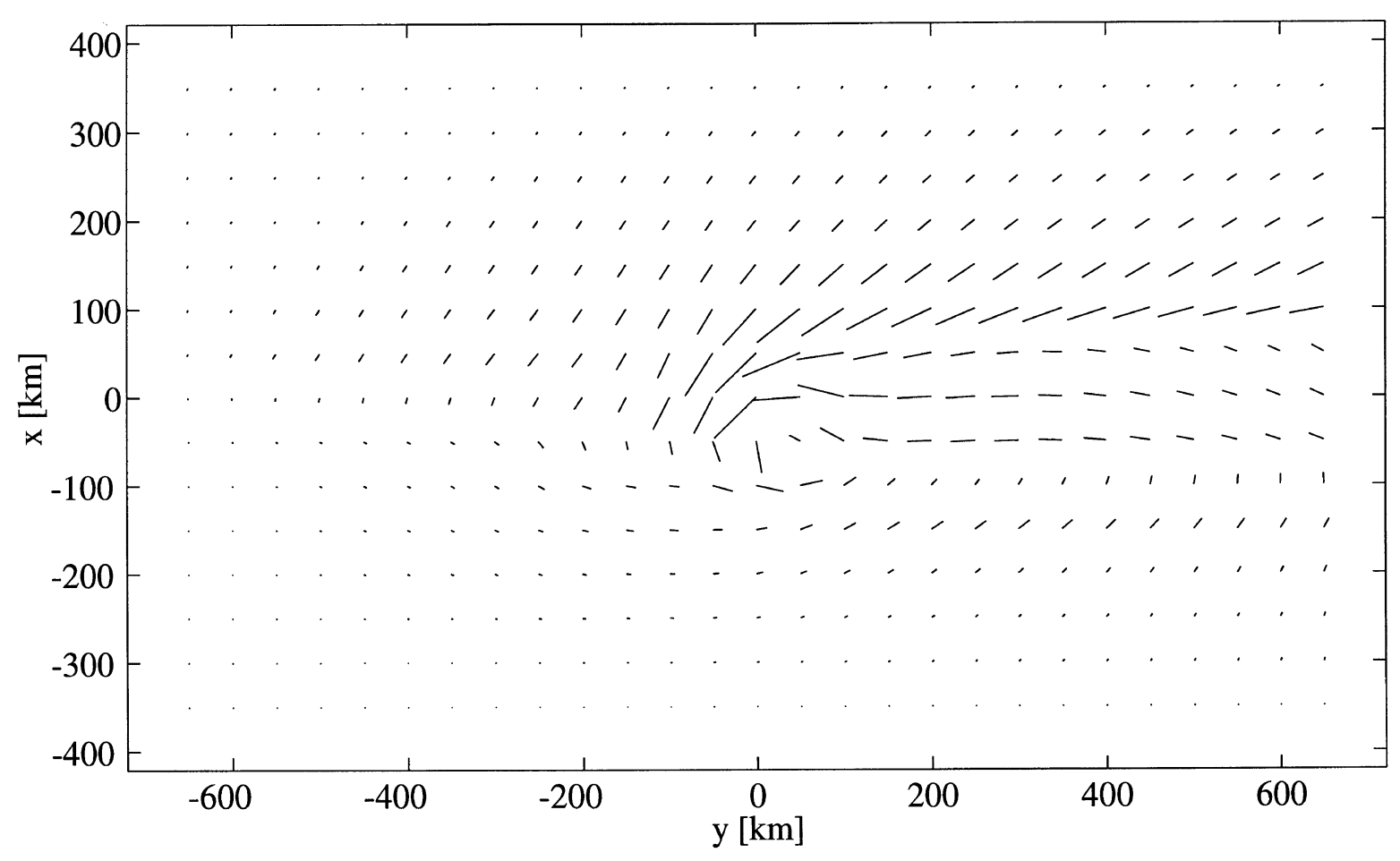

Fig. 5. Horizontal current distribution associated with the westward travelling surge (WTS). This is the snapshot of the instant when the head of WTS at $(x, y)=(0,0)$ is above the origin of the earth surface grid.

\subsection{Harang Discontinuity}

A typical local evening ionospheric current system consists of the boundary between an eastward and a westward electrojet which are separated by an upward current distribution (Fig. 9). We assume that the whole system moves westwards with a velocity of $1 \mathrm{~km} / \mathrm{s}$.

The sounding curves are presented in Figs. 10 and 11. Except for some single spikes, the apparent resistivities are nearly identical to the plane wave ones, when the conductive model is considered. Deviations in the resistive model are clearly not (only) due to such spikes, but have a more systematic behaviour decreasing the apparent resistivities.

\subsection{Omega band}

An omega band is an auroral form which resembles the Greek letter $\Omega$ (Fig. 12). The associated current system moves eastwards with a typical velocity of $1 \mathrm{~km} / \mathrm{s}$. In the time domain a characteristic feature is a positive peak in $B_{y}$ when the current system moves over an observation point.

The sounding curves are presented in Figs. 13 and 14. Their characteristics are comparable to the Harang discontinuity case: deviations from the plane wave results are smaller for the conductive earth model than for the resistive one.

\subsection{Giant pulsation}

A giant pulsation is observed as a nearly sinusoidal time variation of the electromagnetic field. The model used here is based on observations by Glassmeier (1980). The ionospheric current system consists of a horizontal vortex having vertical currents at its centre and opposite vertical currents at both sides (Fig. 15). In this case the system is moving westwards with about the Earth's rotation velocity, and each of the elementary current filaments has an approximately sinusoidal time dependence (period $100 \mathrm{~s}$ ).
Although the ionospheric current is spatially strongly inhomogeneous, the apparent resistivity is nearly equal to that of the plane wave. However, a denser plot of $\rho_{y x}$ along the profile $y=0 \mathrm{~km}$ would reveal some single spikes. In Fig. 16 we only show the resistive case, because for the conductive model the sounding curves of $\rho_{x y}$ and $\rho_{y x}$ are practically identical to the plane wave ones. Although the source effect happens to be small along the selected $y$ profile, it is somewhat more severe at some other profiles. In any case, the apparent resistivity is clearly less distorted than in other models.

\section{Conclusions}

The complex image method (CIM) is a powerful tool to compute the electromagnetic field at the earth's surface produced by realistic 3D auroral ionospheric current systems. Some basic features of the source effect known from previous theoretical investigations are confirmed again in this paper. As a general rule, the source effect diminishes with increasing distance from the centre of the ionospheric current system, and with increasing conductivity of the earth. However, the dependence on the period seems to be more complicated than generally thought: in several models, the source distortion is more severe at small periods than in the longperiod range. The discrepancy between the conductive and resistive earth models used here is quite drastic, although the difference between the corresponding complex skin depths does not appear particularly large. In conclusion, the distortion is much more complicated than considered in earlier works, although some indication of a peculiar behaviour has been pointed out earlier in connection with simple finitelength electrojet models (Häkkinen et al., 1989; Viljanen et 

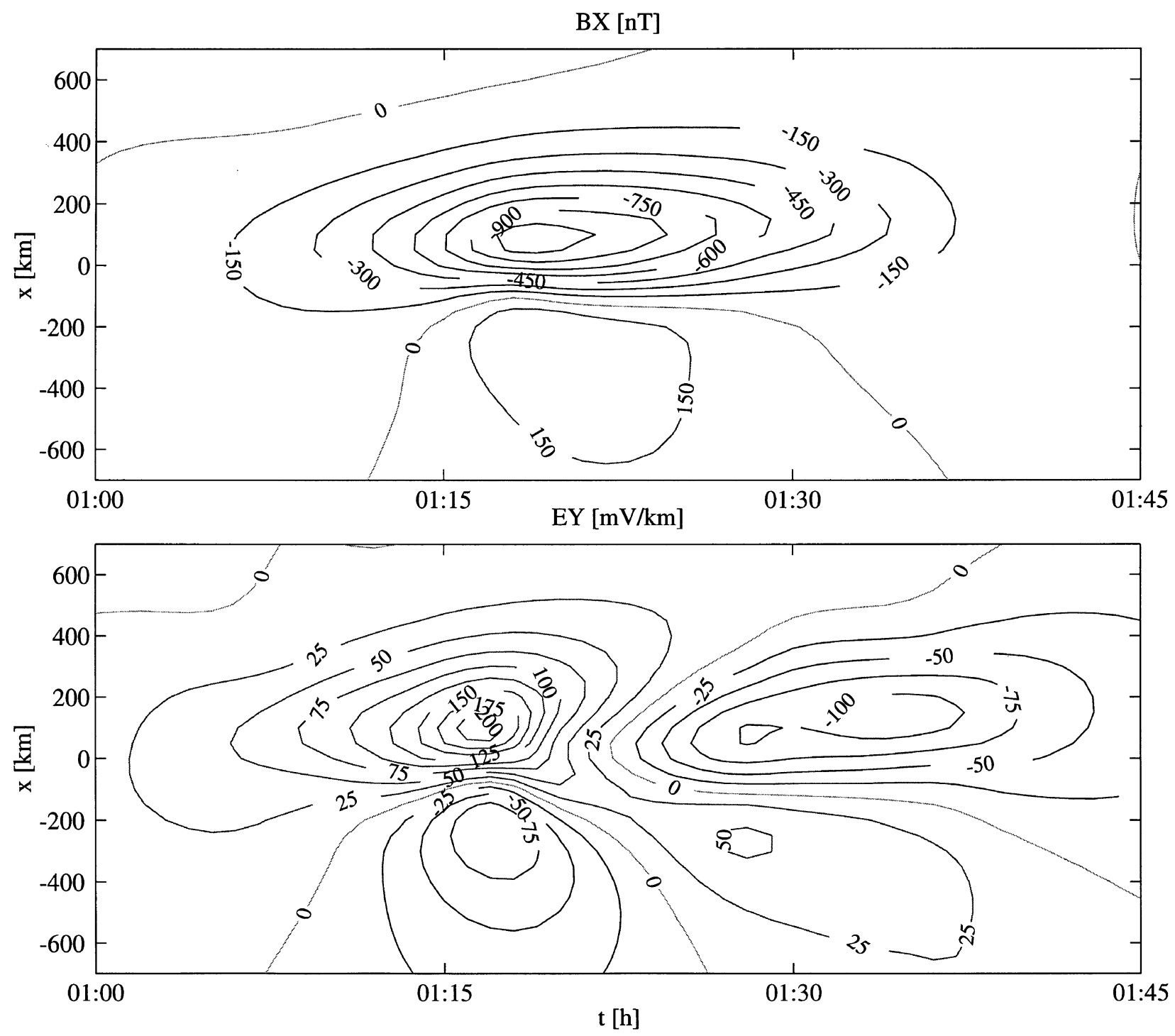

Fig. 6. $B_{x}(x, t)$ and $E_{y}(x, t)$ along the profile $y=0 \mathrm{~km}$ for a WTS moving $1 \mathrm{~km} / \mathrm{s}$ westwards. The conducitive earth model is used.

al., 1993) or in real data (e.g. Garcia et al., 1997).

There are no large differences in the size of distortion between the two apparent resistivities $\left(\rho_{x y}\right.$ and $\left.\rho_{y x}\right)$. However, at long periods $\rho_{x y}$ is generally a little closer to the plane wave value than $\rho_{y x}$. This is evidently due to the fact that in most models the ionospheric current system moves in the east-west direction and thus may produce smoother $\left(E_{x}, B_{y}\right)$ than $\left(B_{x}, E_{y}\right)$. Anyhow, the source effect cannot be directly estimated from the structure of the ionospheric current. For example, due to a giant pulsation the surface field is laterally strongly inhomogeneous, but the apparent resisitivities are nearly identical to the plane wave values.

Our examples concern single events, especially selected to be caused by very inhomogeneous sources. This may give too a pessimistic impression of the source effect. In practical magnetotelluric analysis, long time series and appropriate robust methods are used, so the effect of single peculiar events may well be removed, and naturally there is no reason to consciously select disturbed samples.
Concerning the future work, an important question is whether the small scale variations in the earth's conductivity affect the electric field more than the inhomogeneous source field. It may even turn out that, after all, the local geology is much more dominating than any complicated source field, at least at small periods.

At the moment CIM is restricted to layered earth models. The next step is to include a lateral variation of the earth's conductivity using the thin sheet method (e.g. Vasseur and Weidelt, 1977). As an input, it requires the normal field, which is determined without the thin sheet at the surface of a layered earth. CIM then provides a convenient way to include realistic ionospheric current models. Such a study will be a part of the theoretical investigations included in the BEAR project (Baltic Electromagnetic Array Research) whose measurements were carried out on the Fennoscandian Shield in summer 1998. 

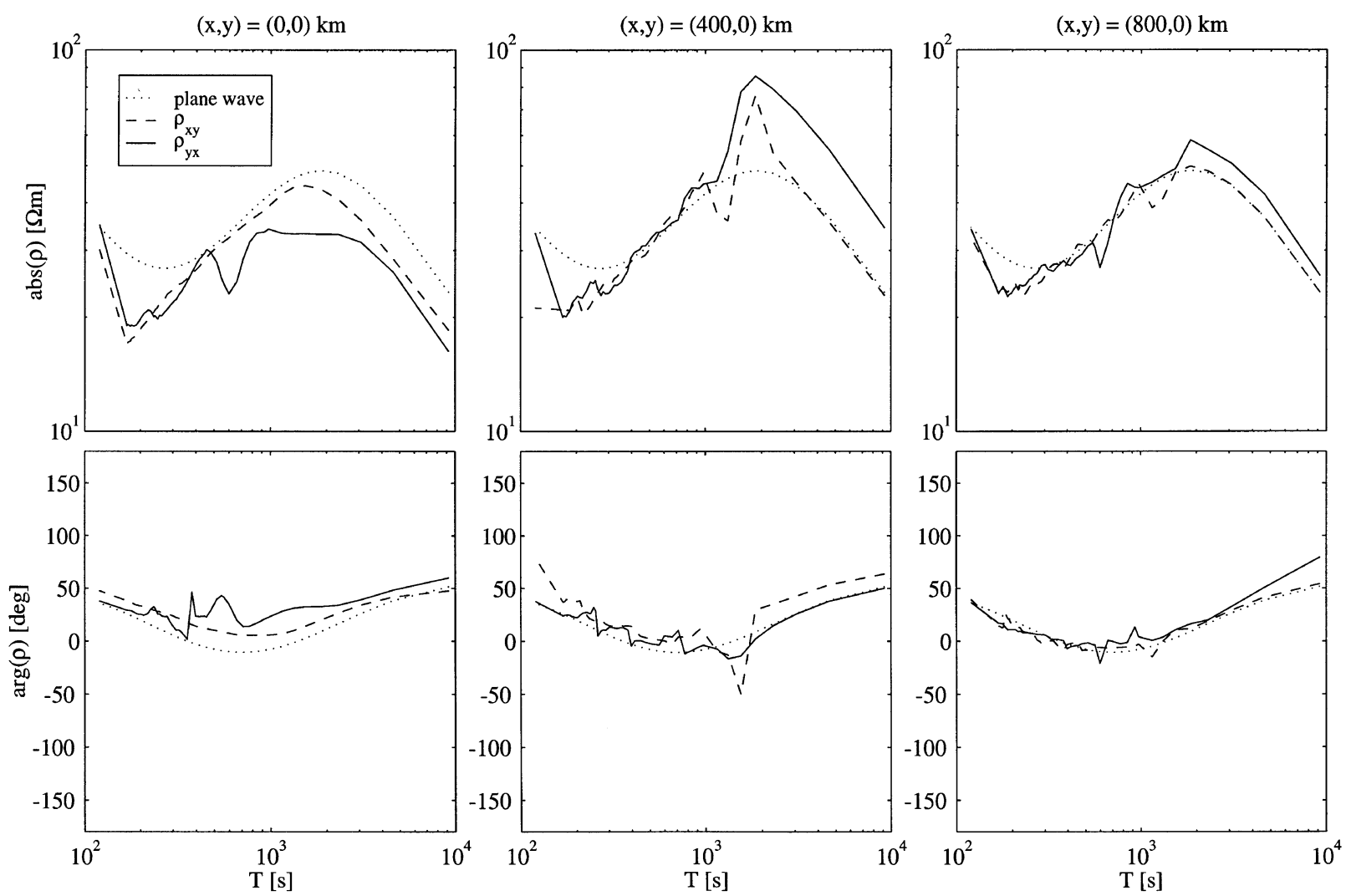

Fig. 7. Absolute values and phases of the apparent resistivities $\rho_{x y}$ and $\rho_{y x}$ as functions of period at $x=0,400,800 \mathrm{~km}(y=0 \mathrm{~km})$ in the case of the WTS model in Fig. 5. The conductive earth model is used. The plane wave value is plotted as a dotted line.
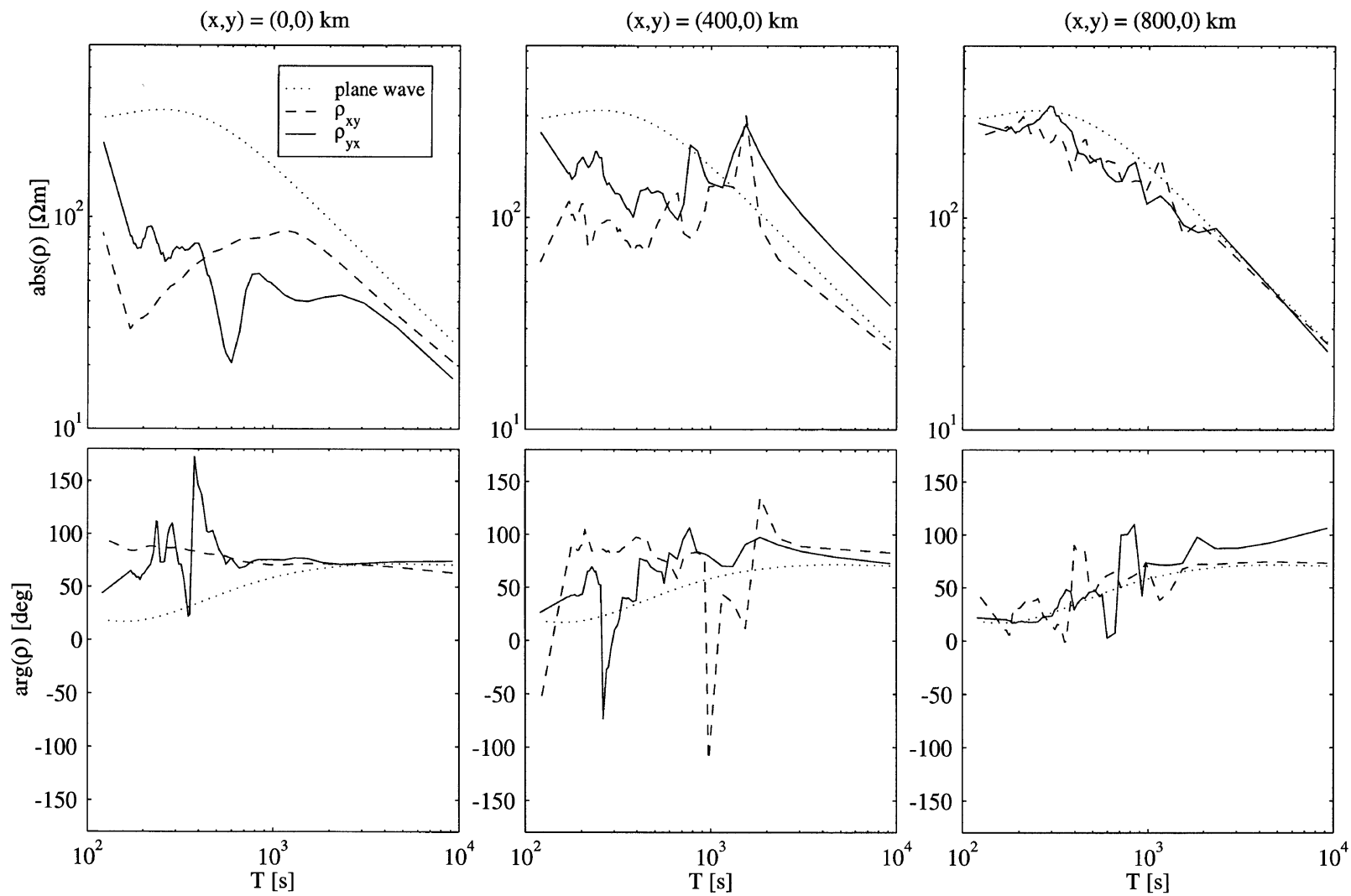

Fig. 8. As Fig. 7, but for the resistive earth model. 


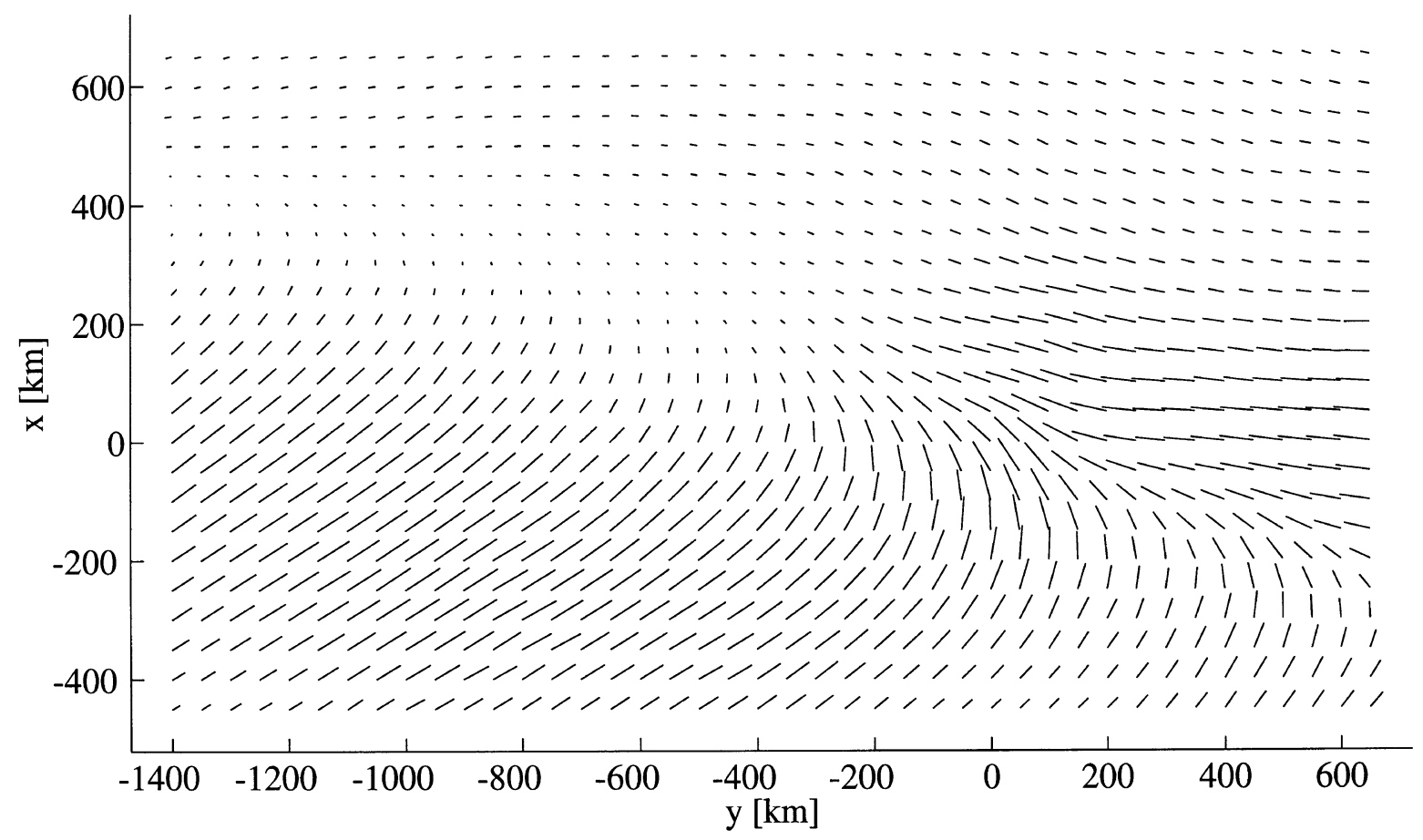

Fig. 9. Snapshot of the horizontal current system of the Harang discontinuity. Along the boundary between the eastward and westward electrojets there is a sharply concentrated upward current sheet approximately along the diagonal from the upper left corner to the lower right corner.
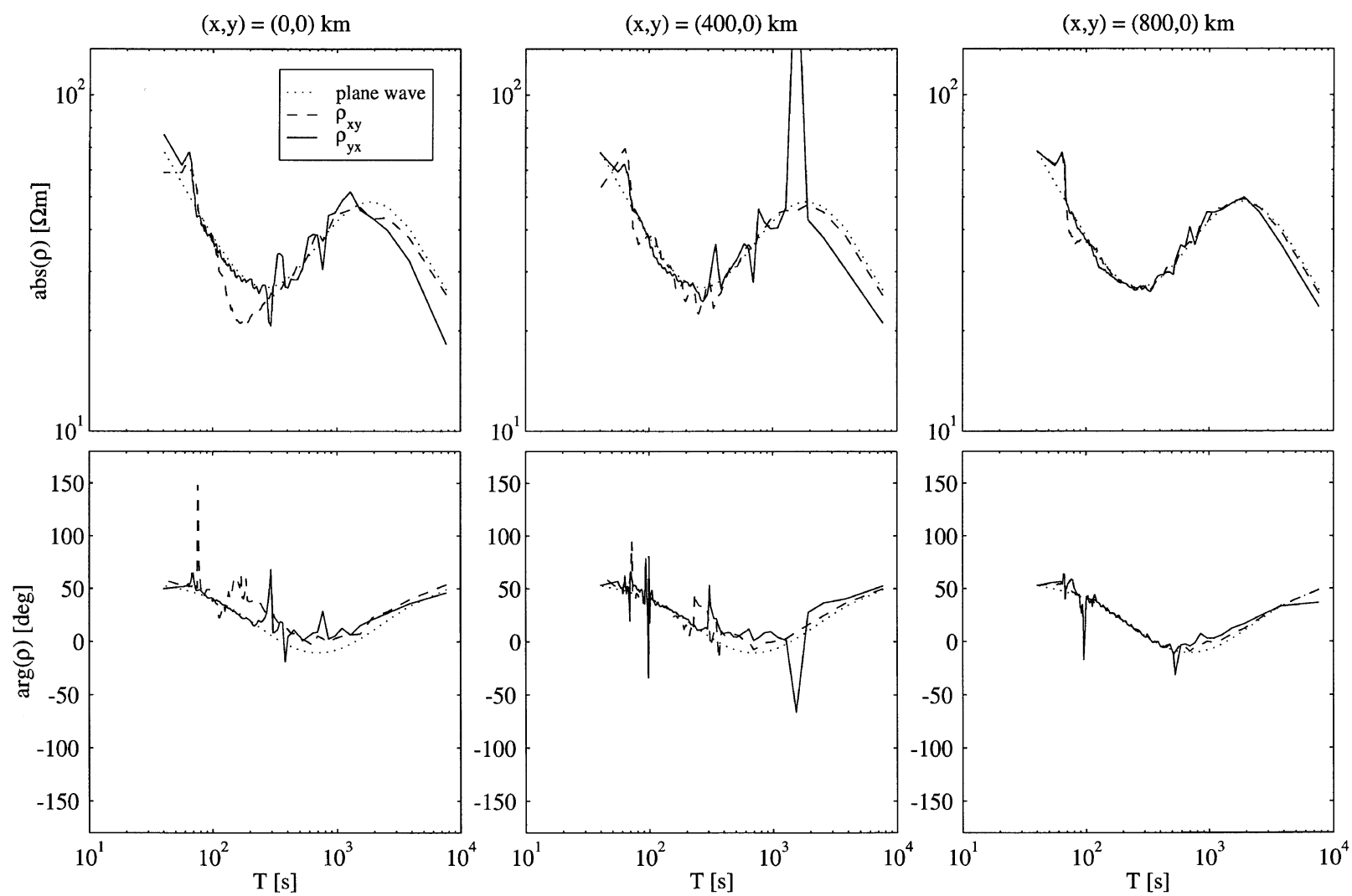

Fig. 10. Absolute values and phases of the apparent resistivities $\rho_{x y}$ and $\rho_{y x}$ as a function of period at $x=0,400,800 \mathrm{~km}(y=0 \mathrm{~km})$ in the case of the Harang discontinuity. The conductive earth model is used. The plane wave value is plotted as a dotted line. 

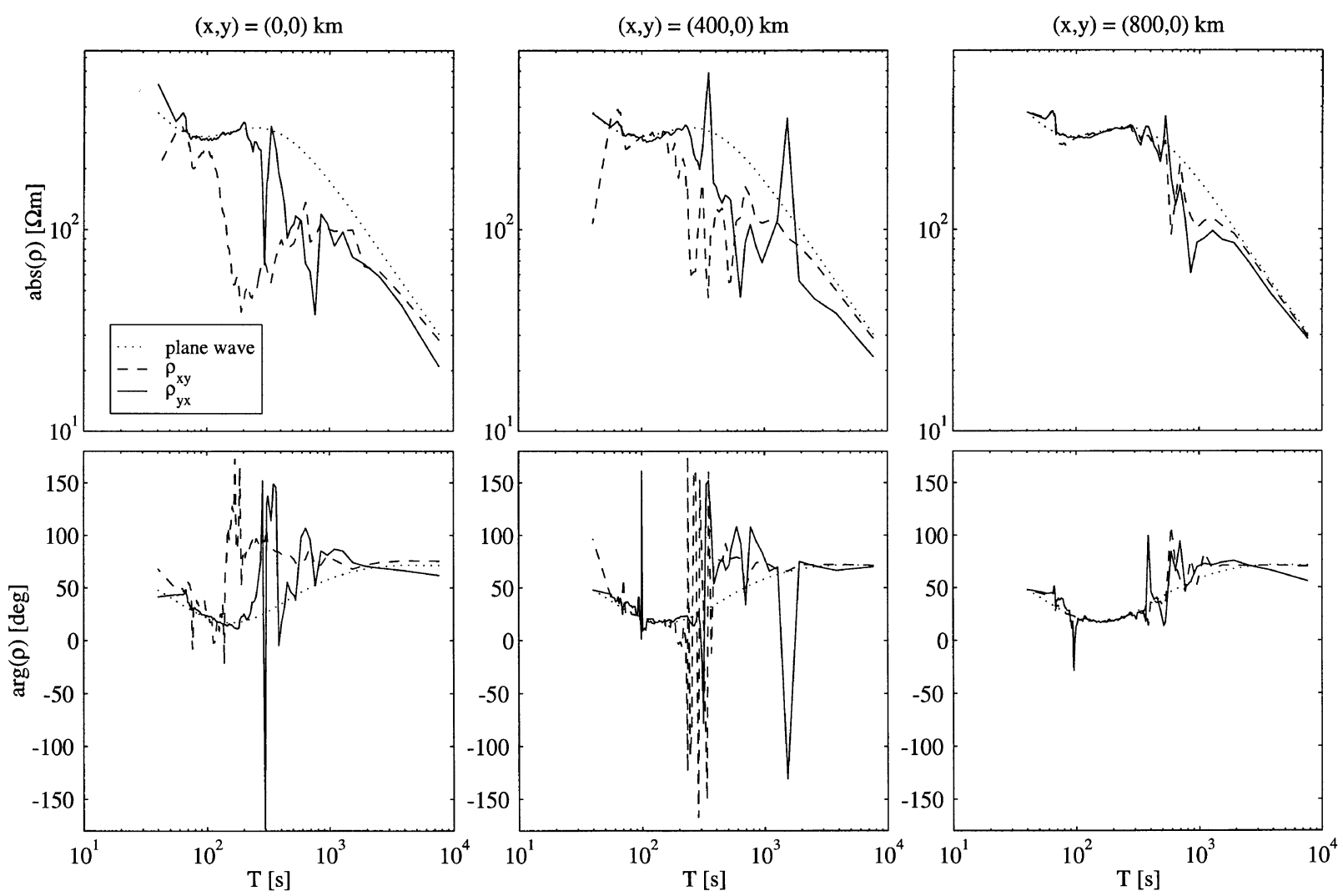

Fig. 11. As Fig. 10, but for the resistive earth model.

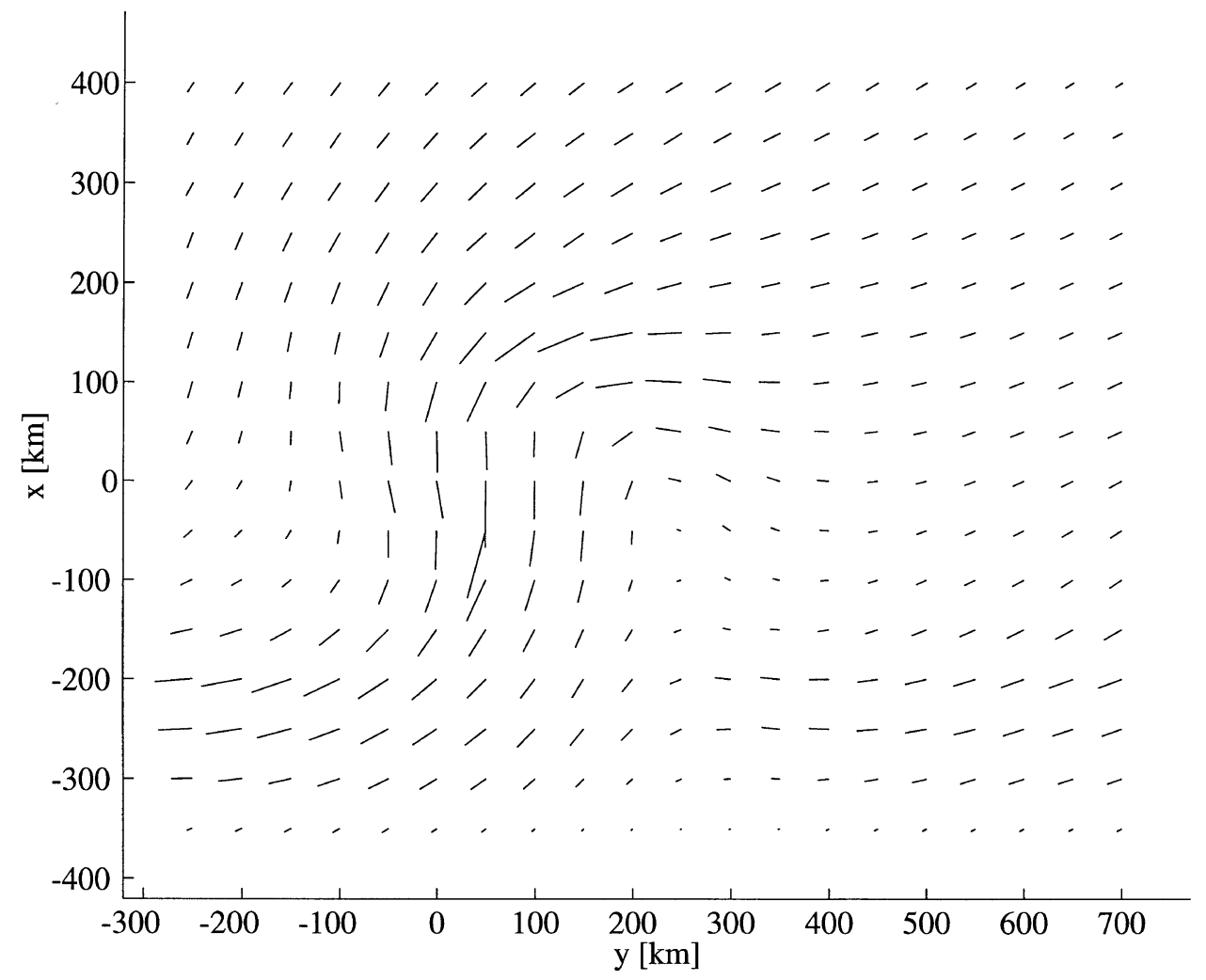

Fig. 12. Snapshot of the horizontal current system of the omega band. 

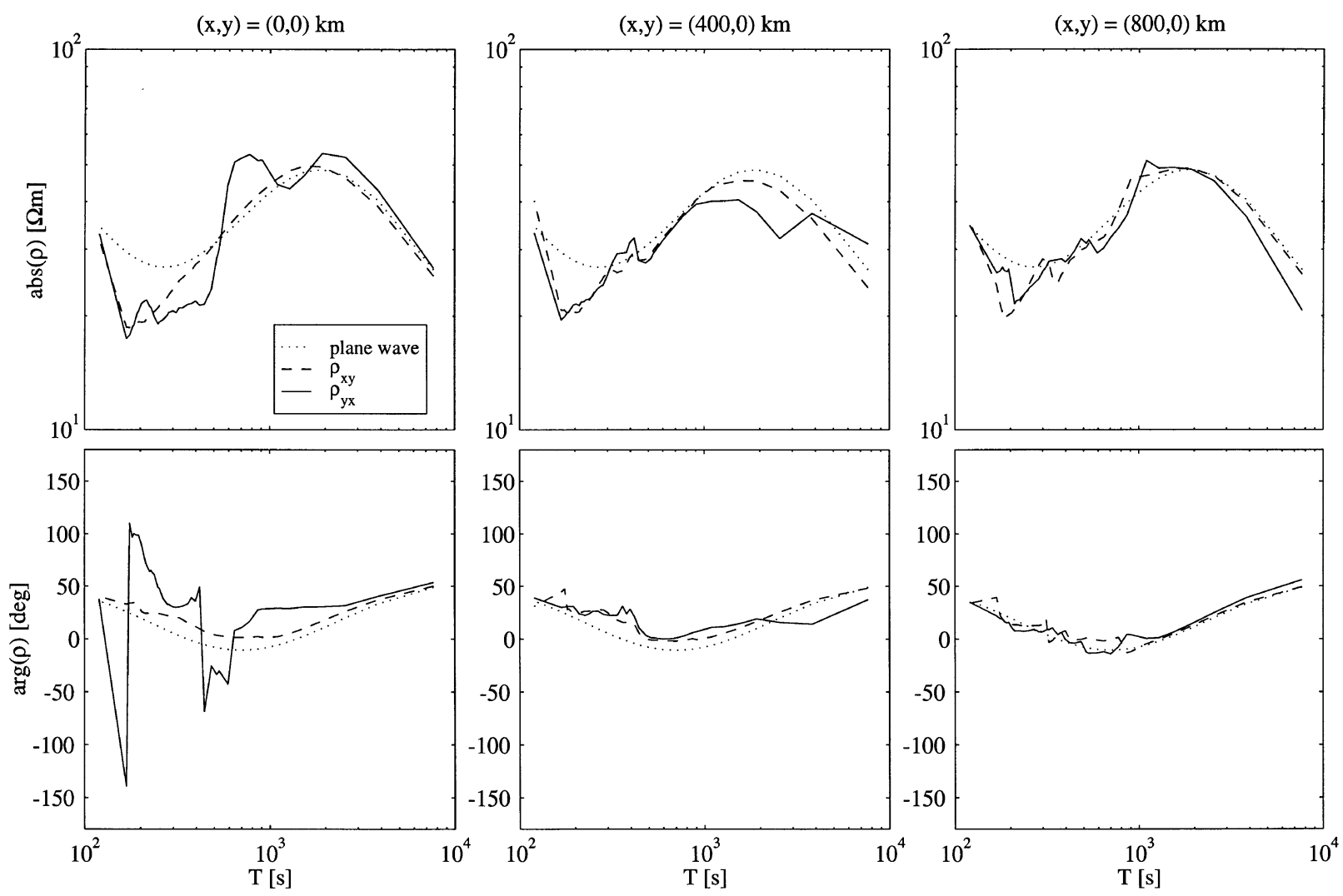

Fig. 13. Absolute values and phases of the apparent resistivities $\rho_{x y}$ and $\rho_{y x}$ as a function of period at $x=0,400,800 \mathrm{~km}(y=0 \mathrm{~km})$ in the case of the omega band. The conductive earth model is used. The plane wave value is plotted as a dotted line.
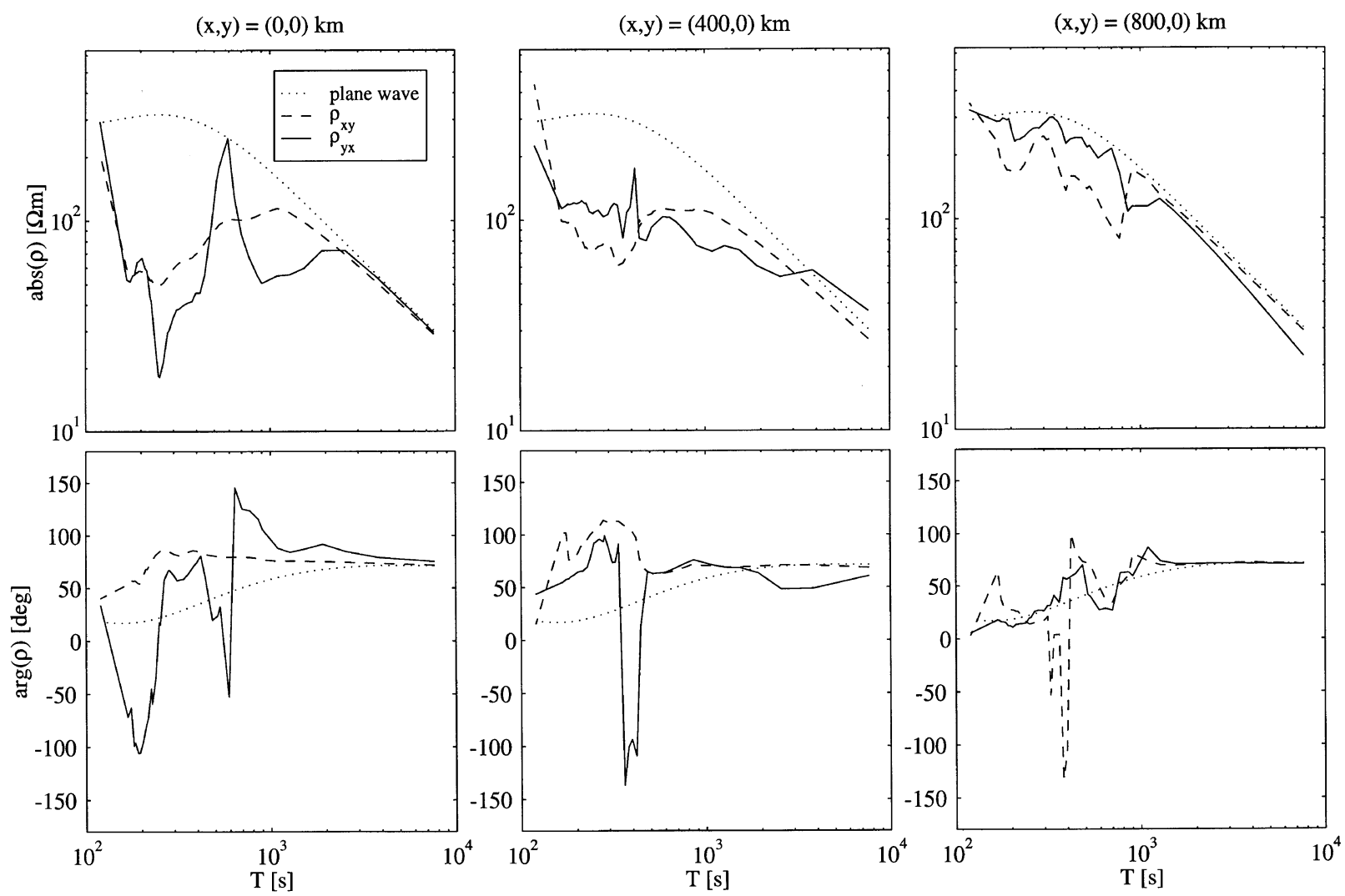

Fig. 14. As Fig. 13, but for the resistive earth model. 


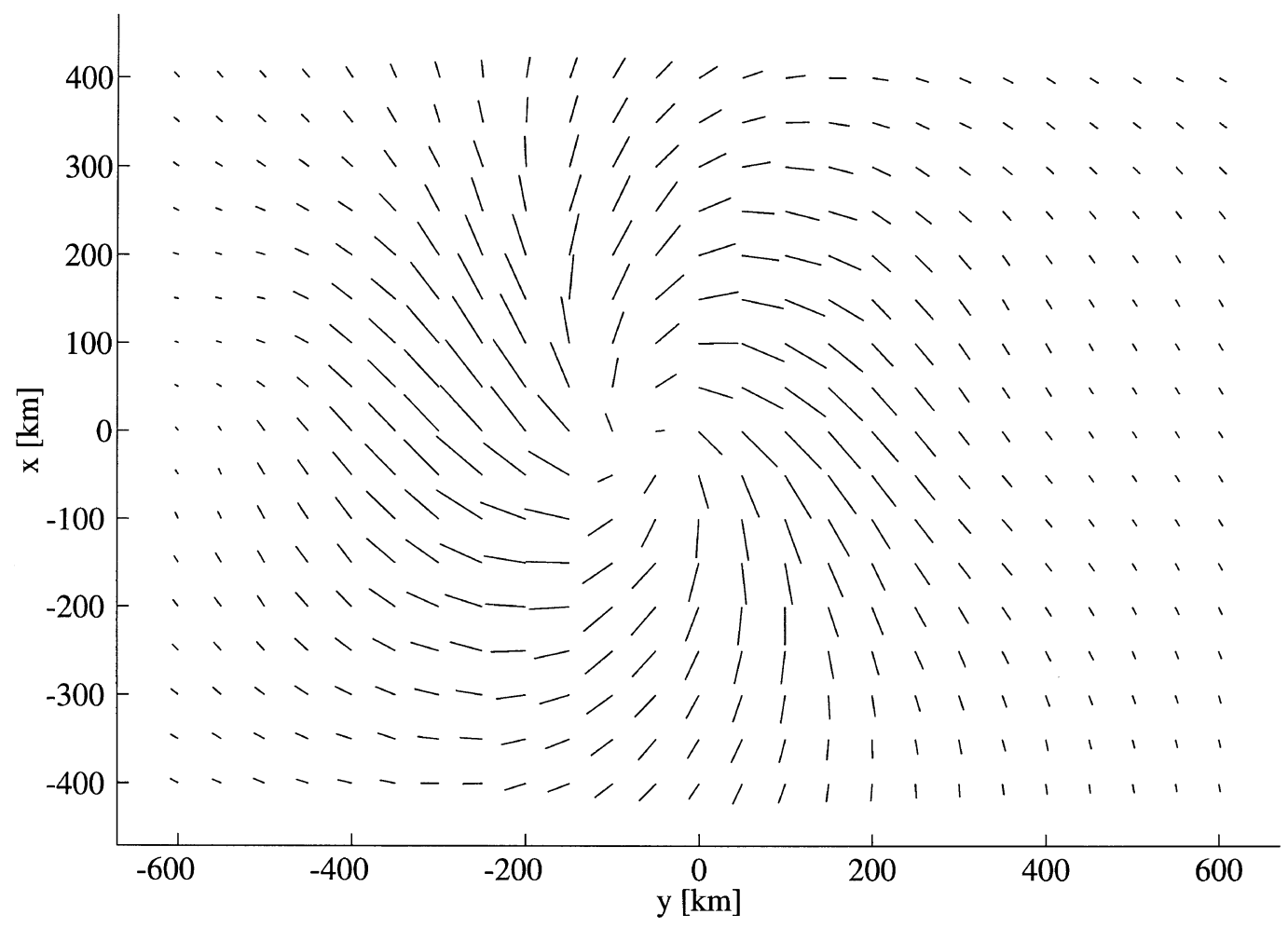

Fig. 15. Snapshot of the horizontal current system producing giant pulsations.
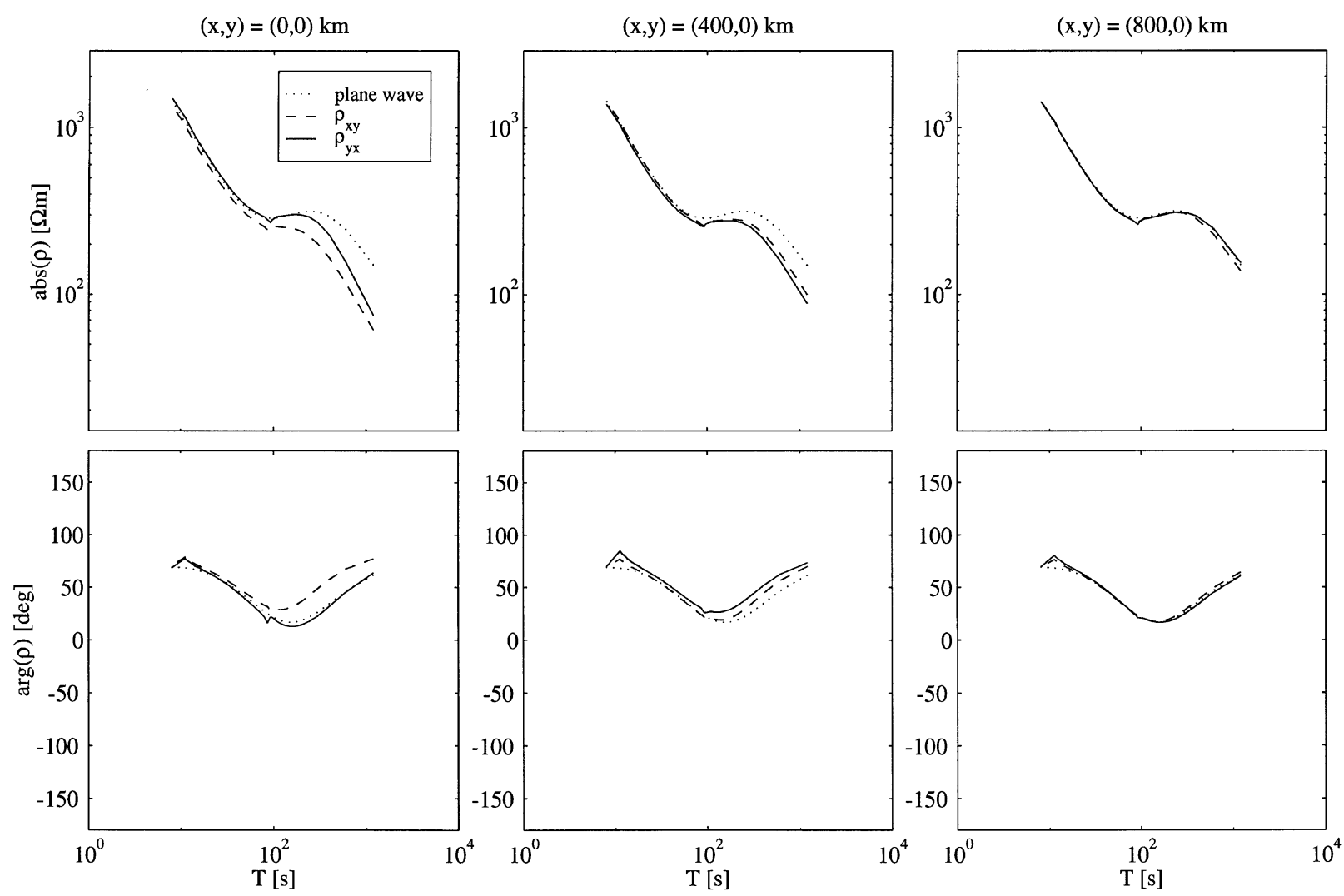

Fig. 16. Absolute values and phases of the apparent resistivities $\rho_{x y}$ and $\rho_{y x}$ as a function of period at $x=0,400,800 \mathrm{~km}(y=0 \mathrm{~km})$ in the case of the giant pulsation. The resistive earth model is used. The plane wave value is plotted as a dotted line. 


\section{Appendix. Field of an Eastward Elementary Cur- rent}

Consider an electrojet of a finite length, having vertical currents at both ends. Assume that the horizontal current flows from $\left(x_{0}, y_{0},-h\right)$ to $\left(x_{0}, y_{1},-h\right)$, a downward current ends at $\left(x_{0}, y_{0},-h\right)$, and an upward current starts at $\left(x_{0}, y_{1},-h\right)$. The amplitude of the current is $I$, and the angular frequency is $\omega$.

The complex image depth is (Boteler and Pirjola, 1998; Pirjola and Viljanen, 1998)

$$
p=p(\omega)=\frac{Z(\omega)}{i \omega \mu_{0}}
$$

where the plane wave surface impedance $Z$ can be calculated from a recursion formula (cf. Wait, 1981, pp. 52-53).

The primary magnetic and electric fields at the earth's surface due to the horizontal current filament are

$$
\begin{aligned}
& B_{x p}(x, y)=-\frac{\mu_{0} I h}{4 \pi r_{1}^{2}}\left(\frac{y-y_{1}}{\sqrt{r_{1}^{2}+\left(y-y_{1}\right)^{2}}}\right. \\
& \left.-\frac{y-y_{0}}{\sqrt{r_{1}^{2}+\left(y-y_{0}\right)^{2}}}\right) \\
& B_{z p}(x, y)=-\frac{\mu_{0} I\left(x-x_{0}\right)}{4 \pi r_{1}^{2}}\left(\frac{y-y_{1}}{\sqrt{r_{1}^{2}+\left(y-y_{1}\right)^{2}}}\right. \\
& \left.-\frac{y-y_{0}}{\sqrt{r_{1}^{2}+\left(y-y_{0}\right)^{2}}}\right) \\
& E_{y p}(x, y)=\frac{i \omega \mu_{0} I}{4 \pi} \\
& \cdot \ln \frac{-y+y_{0}+\sqrt{\left(x-x_{0}\right)^{2}+\left(y-y_{0}\right)^{2}+h^{2}}}{-y+y_{1}+\sqrt{\left(x-x_{0}\right)^{2}+\left(y-y_{1}\right)^{2}+h^{2}}}
\end{aligned}
$$

where $r_{1}^{2}=\left(x-x_{0}\right)^{2}+h^{2} . \quad B_{y p}$ and $E_{x p}$ are zero. The secondary fields are obtained by replacing $h$ with $h+2 p$, and changing the sign of $B_{z p}$ and $E_{y p}$.

The primary field due to the radial horizontal current that is equivalent to the downward current at $\left(x_{0}, y_{0},-h\right)$ is

$$
\begin{gathered}
B_{x p}(x, y)=\frac{\mu_{0} I\left(y-y_{0}\right)}{4 \pi r_{2}^{2}}\left(-1+\frac{h}{\sqrt{r_{2}^{2}+h^{2}}}\right) \\
B_{y p}(x, y)=\frac{\mu_{0} I\left(x-x_{0}\right)}{4 \pi r_{2}^{2}}\left(-1+\frac{h}{\sqrt{r_{2}^{2}+h^{2}}}\right) \\
E_{x p}(x, y)=\frac{i \omega \mu_{0} I}{4 \pi} \frac{x-x_{0}}{\sqrt{r_{2}^{2}+h^{2}}+h} \\
E_{y p}(x, y)=\frac{i \omega \mu_{0} I}{4 \pi} \frac{y-y_{0}}{\sqrt{r_{2}^{2}+h^{2}}+h}
\end{gathered}
$$

where $r_{2}^{2}=\left(x-x_{0}\right)^{2}+\left(y-y_{0}\right)^{2}$ (for a detailed derivation, see Pirjola and Viljanen, 1998). $B_{z p}$ is zero. The secondary field is obtained by replacing $h$ with $h+2 p$, and changing the sign of the electric field. Formulas for the upward current at $\left(x_{0}, y_{1},-h\right)$ are obtained similarly by replacing $\left(x_{0}, y_{0}\right)$ with $\left(x_{0}, y_{1}\right)$ and changing the signs.

Formulas for the fields produced by a northward filament are obtained in an obvious manner from Eqs. (A.2) and (A.3) by a rotation of the coordinate system.

\section{References}

Amm, O., Direct determination of the local ionospheric Hall conductance distribution from two-dimensional electric and magnetic field data: Application of the method using models of typical ionospheric electrodynamic situations, J. Geophys. Res., 100(A11), 21473-21488, 1995.

Amm, O., Improved electrodynamic modeling of an omega band and analysis of its current system, J. Geophys. Res., 101, 2677-2683, 1996.

Boteler, D. H. and R. J. Pirjola, The complex-image method for calculating the magnetic and electric fields at the surface of the Earth by the auroral electrojet, Geophys. J. Int., 132, 31-40, 1998.

Chave, A. D., D. J. Thomson, and M. E. Ander, On the robust estimation of power spectra, coherences, and transfer functions, J. Geophys. Res., 92, 633-648, 1987.

Egbert, G. D. and J. R. Booker, Multivariate analysis of geomagnetic array data. 1. The response space, J. Geophys. Res., 94, 14227-14247, 1989a

Egbert, G. D. and J. R. Booker, Multivariate analysis of geomagnetic array data. 2. Random source models, J. Geophys. Res., 94, 14249-14265, $1989 b$.

Garcia, X., A. D. Chave, and A. G. Jones, Robust processing of magnetotelluric data from the auroral zone, J. Geomag. Geoelectr., 49, 1451-1468, 1997.

Glassmeier, K.-H., Magnetometer array observations of a giant pulsation event, J. Geophys., 48, 127-138, 1980

Häkkinen, L. and R. Pirjola, Calculation of electric and magnetic fields due to an electrojet current system above a layered earth, Geophysica, 22, 31-44, 1986.

Häkkinen, L., R. Pirjola, and C. Sucksdorff, EISCAT magnetometer cross and theoretical studies connected with the electrojet current system, Geophysica, 25, 123-134, 1989.

Korja, T. and the BEAR Working Group, BEAR. Baltic Electromagnetic Array Research, EUROPROBE News, No. 12, 4-5, 1998.

Larsen, J. C., R. L. Mackie, A. Manzella, A. Fiordelisi, and S. Rieven, Robust smooth magnetotelluric transfer function, Geophys. J. Int., 124, 801-819, 1996

Mareschal, M., Modelling of natural sources of magnetospheric origin in the interpretation of regional induction studies: a review, Surv. Geophys., 8, 261-300, 1986.

Osipova, I. L., S. E. Hjelt, and L. L. Vanyan, Source field problems in northern parts of the Baltic Shield, Phys. Earth Planet. Inter, 53, $337-$ 342, 1989.

Padilha, A. L., I. Vitorello, and L. Rijo, Effects of the Equatorial Electrojet on magnetotelluric surveys: Field results from Northwest Brazil, Geophys. Res. Lett., 24, 89-92, 1997.

Pirjola, R., On magnetotelluric source effects caused by an auroral electrojet system, Radio Sci., 27(4), 463-468, 1992.

Pirjola, R. and A. Viljanen, Complex image method for calculating electric and magnetic fields produced by an auroral electrojet of a finite length Ann. Geophys., 16, 1434-1444, 1998.

Pirjola, R., D. Boteler, A. Viljanen, and O. Amm, Prediction of geomagnetically induced currents in power transmission systems, Adv. Space Res., 1999 (accepted for publication).

Szarka, L. and G. Fischer, Electromagnetic parameters at the surface of a conductive halfspace in terms of the subsurface current distribution, Geophysical Transactions, 25(3), 157-172, 1989.

Thomson, D. J. and J. T. Weaver, The complex image approximation for induction in a multilayered Earth, J. Geophys. Res., 80(1), 123-129, 1975.

Untiedt, J. and W. Baumjohann, Studies of polar current systems using the IMS Scandinavian magnetometer array, Space Science Reviews, 63, 245-390, 1993.

Vasseur, G. and P. Weidelt, Bimodal electromagnetic induction in nonuniform thin sheets with an application to the northern Pyrenean induction anomaly, Geophys. J. R. astr. Soc., 51, 669-690, 1977. 
Viljanen, A., Source effect on geomagnetic induction vectors in the fennoscandian auroral region, J. Geomag. Geoelectr., 48, 1001-1009, 1996.

Viljanen, A. and R. Pirjola, On the possibility of performing studies on the geoelectric field and ionospheric currents using induction in power systems, J. Atmos. Terr. Phys., 56, 1483-1491, 1994.

Viljanen, A., R. Pirjola, and L. Häkkinen, An attempt to reduce induction source effects at high latitudes, J. Geomag. Geoelectr., 45, 817-831, 1993.

Wait, J. R., Wave Propagation Theory, 348 pp., Pergamon Press, 1981.
Wait, J. R. and K. P. Spies, On the representation of the quasi-static fields of a line current source above the ground, Can. J. Phys., 27, 2731-2733, 1969.

Weidelt, P., The Inverse Problem of Geomagnetic Induction, Z. Geophys., 38, 257-289, 1972 .

A. Viljanen (e-mail: ari.viljanen@fmi.fi), R. Pirjola (e-mail: risto. pirjola@fmi.fi), and O. Amm (e-mail: olaf.amm@fmi.fi) 